\title{
Guest editorial: Special issue on crowd sensing networks
}

\author{
Mianxiong Dong ${ }^{1} \cdot$ Fen $\mathrm{Hou}^{2} \cdot$ Peng Cheng ${ }^{3} \cdot$ Kyoung-Sook Kim ${ }^{4}$
}

Published online: 31 March 2016

(C) Springer Science+Business Media New York 2016

With the miniaturization of sensors, we have been witnessing the popularity of the sensor-build-in mobile devices over the past few years. This creates a new trend for next generation sensor networks: the crowd sensing networks (CSNs), which is expected to provide a promising platform for the acquiring of ubiquitous and participatory sensing data. With the involvement of a huge number of mobile users or other devices such as sensor-equipped vehicles, the crowd sensing networks can provide a lot of novel and valuable applications such as environmental monitoring and protection, traffic jam alternatives, citizen-journalism, tourist query, wireless indoor localization, etc.

Although there have been remarkable developments in the field of crowd sensing, we still face many challenging issues that need to be addressed in order to achieve the success of crowd

Mianxiong Dong

mx.dong@csse.muroran-it.ac.jp

Fen Hou

fenhou@umac.mo

Peng Cheng

pcheng@iipc.zju.edu.cn

Kyoung-Sook Kim

ks.kim@aist.go.jp

1 Muroran Institute of Technology, Muroran, Hokkaido 050-8585, Japan

2 University of Macau, Avenida da Universidade, Taipa, Macau 999078, China

3 Zhejiang University, Hangzhou, Zhejiang 310027, China

4 National Institute of Advanced Industrial Science and Technology (AIST), Koto-ku, Tokyo 135-0064, Japan sensing, e.g. how to optimize the routing scheme to improve the reliability and performance of the CSNs with limited battery and memory; how to design incentive mechanism to motivate mobile users to participate the sensing work; how to resist attacks and protect the privacy of the CSNs users, etc. These problems can be even more severe with the emerging new applications.

This Special Issue on CSNs devoted to recent addressing these challenges. A Call for Papers was issued in early 2014, with the submission deadline set to November 1st, 2014. We have received an overwhelming number of submissions, and ultimately 14 high quality papers were selected for this special issue.

The paper "A reliable QoS-aware routing scheme for neighbor area network in smart grid", authored by Xiaoheng Deng, Lifang $\mathrm{He}, \mathrm{Xu} \mathrm{Li}$, Qiang Liu, Lin Cai, and Zhigang Chen, proposes a neighbor area network routing scheme to meet the QoS requirements of different smart grid applications, and a routing reliability optimization method to further improve the network reliability.

The paper "Multi-hop Data Forwarding Method for Crowd Sensing Networks", authored by Yeonsu Jung and Yunju Baek, focuses on the CSNs with limitation of energy consumption and memory usage. The authors propose a lightweight multi-hop data forwarding method, and adopt a novel address abbreviation method to handle the packet fragmentation problem resulted from utilizing DSR over IEEE 802.15.4.

The paper "CLEVER: cluster-based energy-aware virtual ring routing in randomly deployed wireless sensor networks", authored by Ghofrane Fersi, Wassef Louati, and Maher Ben Jemaa, presents another novel routing protocol for the networks with energy-constrained nodes. Compared to existing approaches, the proposed method shows a significant improvement in the network lifetime and energy consumption.

The paper "Perceiving who and when to leverage data delivery for maritime networks: an optimal stopping view", 
authored by Tingting Yang, Chengming Yang, Hailong Feng, and Ruilong Deng, investigates the data scheduling issue in Maritime CSNs, adopting store-carry-and-forward protocol in DTNs. A Two-step Time and Energy Oriented Optimalstopping algorithm leveraging backward induction method is proposed, based on the optimal stopping rules.

The paper "Performance improvement for source mobility in named data networking based on global-local FIB updates", authored by Jingguo Ge, Shaojie Wang, Yulei Wu, Haina Tang, and Yuepeng E, proposes a new packet forwarding algorithm to perform fast Forwarding Information Base updates of Named Data Networking nodes based on Software Defined Networking paradigm with low control overhead, which outperforms the existing solutions based on ID-Locator split architecture.

The paper "Congestion control in social-based sensor networks: a social network perspective", authored by Kaimin Wei, Song Guo, Xiangli Li, Deze Zeng and $\mathrm{Ke} \mathrm{Xu}$, aims to develop an efficient congestion control approach from the social network perspective. The authors propose a multi-attribute decision making model to determine the forwarding set and transmission order, and also a new buffer management scheme.

The paper "An incentive game based evolutionary model for crowd sensing networks", authored by Xiao Liu, Kaoru Ota, Anfeng Liu, and Zhigang Chen, discusses the collaborative game phenomenon of the CSNs. An evolutionary model is proposed to provide decision supports for managers. Their studies are interesting and comprehensive. Simulations support the claims.

The paper "Analytical model with a novel selfishness division of mobile nodes to participate cooperation", authored by Qichao Xu, Zhou Su, Bo Han, Dongfeng Fang, Zejun Xu, and Xiaoying Gan, presents an analytical model for modeling and understanding the effect of mobile individuals' selfishness, in order to encourage the individuals to participate the cooperation of CSNs.

The paper "Duration of stay based weighted scheduling framework for mobile phone sensor data collection in opportunistic crowd sensing", authored by Thejaswini M, P. Rajalakshmi, U. B. Desai, refers to a weighted scheduling framework for collecting mobile phone sensor data under Levy Walk mobility model. The proposed method uses the dwell time of mobile phone users as an important parameter to reduce the miss rate of mobile nodes.

The paper "Top-k query processing in mobile-P2P networks using economic incentive schemes", authored by Nilesh Padhariya, Anirban Mondal, and Sanjay Kumar Madria, proposes a Top-k query processing system issuing economic rewards or penalties to the mobile peers, and several different economic incentive schemes, in which mobile peers act individually towards Top-k query processing.

The paper "An optimal query strategy for protecting location privacy in location-based services", authored by Wei- dong Yang, Yun-hua He, Li-min Sun, Xiang Lu, and Xinghua Li, studies the location privacy from a gametheoretic perspective. An optimal game-theoretic query strategy is presented to resist the tracing and re-identifying attacks, and help users achieve optimized utilities.

The paper "Practical blacklist-based anonymous authentication scheme for mobile crowd sensing", authored by Hongwei Li, Kun Jia, Haomiao Yang, Dongxiao Liu, and Liang Zhou, also focuses on the security and privacy problems in CSNs. Security analysis shows the proposed scheme can achieve anonymity, blacklist ability, nonrepudiation and unlinkability. Simulations have been conducted to support the studies.

The paper "Secure and efficient fine-grained multiple file sharing in cloud-assisted crowd sensing networks", authored by Jun Zhou, Zhenfu Cao, and Xiaolei Dong, proposes a generalized efficient batch cryptosystem to simultaneously achieve batch encryption/decryption from any public key encryption algorithm, and its extensions to address secure multiple file sharing in the CSNs.

The paper "The implementation of indoor localization based on an experimental study of RSSI using a wireless sensor network", authored by Cheriet Mohammed El Amine, Ouslim Mohamed, and Belaidi Boualam, performs an indoor localization application using three algorithms based on the Received Signal Strength Indication fingerprinting. Several experiments have been conducted to prove its effectiveness.

In conclusion, the papers presented in this special issue demonstrate the breadth and diversity of research in the field of CSNs. We wish to thank both the authors and the reviewers for their hard work in helping us organize this special issue. We would also like to express our sincere gratitude to the Editor-in-Chief, Professor Sherman Shen for providing this opportunity and lots of guidance throughout the process, and the editorial staffs of PPNA for their continuous support and professionalism.

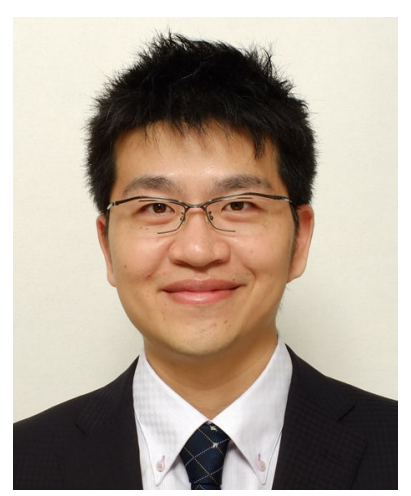

Mianxiong Dong received B.S., M.S. and Ph.D. in Computer Science and Engineering from The University of Aizu, Japan. $\mathrm{He}$ is currently an Associate Professor in the Department of Information and Electronic Engineering at the Muroran Institute of Technology, Japan. Prior to joining Muroran-IT, he was a Researcher at the National Institute of Information and Communications Technology (NICT), Japan. He was a JSPS Research Fellow with School of Computer Science and Engineering, The University of Aizu, Japan and was a visiting scholar with BBCR group at University of Waterloo, Canada supported by JSPS Excellent Young Researcher Overseas Visit 
Program from April 2010 to August 2011. Dr. Dong was selected as aForeigner Research Fellow (a total of 3 recipients all over Japan) by NEC C\&C Foundation in 2011. His research interests include Wireless Networks, Cloud Computing, and Cyber-physical Systems. His research results have been published in 130 research papers in international journals, conferences and books. He has received best paper awards from IEEE HPCC 2008, IEEE ICESS 2008, ICA3PP 2014, GPC 2015, and IEEE DASC 2015. Dr. Dong serves as an Associate Editor for IEEE Communications Surveys and Tutorials, IEEE Network, IEEE Access, and Cyber-Physical Systems (Taylor \& Francis). He is currently a research scientist with A3 Foresight Program (2011-2016) funded by Japan Society for the Promotion of Sciences (JSPS), NSFC of China, and NRF of Korea.

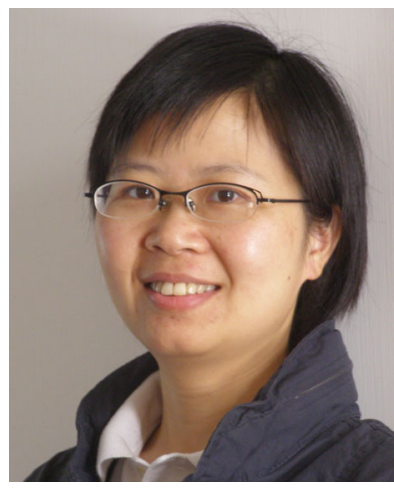

Fen Hou is an Assistant Professor in the Department of Electrical and Computer Engineering at the University of Macau. She received the Ph.D. degree in electrical and computer engineering from the University of Waterloo, Waterloo, Canada, in 2008. Her research interests include resource allocation and protocol design in vehicular sensor network and participatory sensor networks, QoS provisioning for multimedia communications in cognitive radio network and broadband wireless networks. She is the recipient of IEEE GLOBECOM Best Paper Award in 2010 and the Distinguished Service Award in IEEE MMTC in 2011. Dr. Fen Hou has served as a Co-Chair in ICCS 2014 Special Session on Economic Theory and Communication Networks, co-chair in INFOCOM 2014 Workshop on Green Cognitive Communications and Computing Networks (GCCCN), and co-chair in IEEE Globecom Workshop on Cloud Computing System, Networks, and Application (CCSNA) 2013 and 2014, respectively. She served as a technical program committee member for IEEE INFOCOM, WiOpt, ICC, WCNC, Globecom, etc.

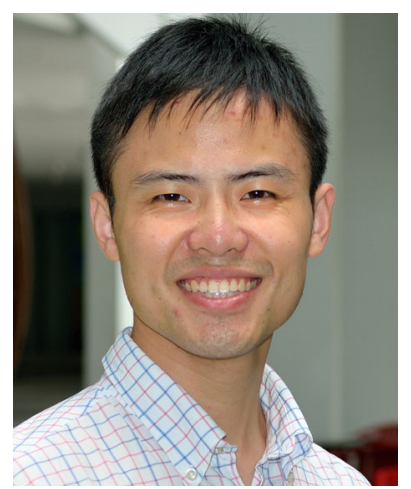

Peng Cheng received the B.E. degree in automation and the Ph.D. degree in control science and engineering from Zhejiang University, Hangzhou, China, in 2004 and 2009 , respectively. He is currently Professor with the College of Control Science and Engineering, Zhejiang University. His current research interests include networked sensing and control, cyber-physical systems, and control system security. Prof. Cheng serves/has served as Associate Editor of Wireless Networks, International Journal of Communication Systems, and Guest Editor of the IEEE Transactions on Control of Network Systems. He served as a Local Arrangement Chair of the IEEE International Symposium on Mobile Ad Hoc Networking and Computing in 2015, and the Publicity Co-Chair of the IEEE Mobile Ad hoc and Sensor Systems in 2013.

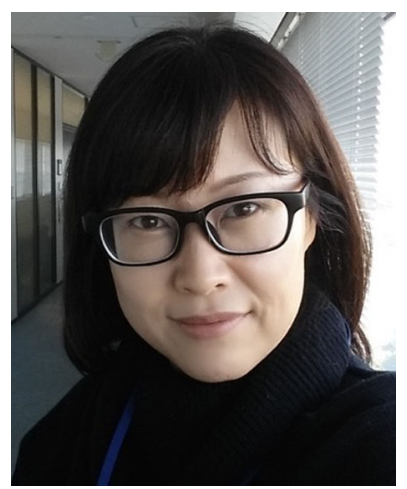

Kyoung-Sook Kim is a researcher of National Institute of Advanced Industrial Science and Technology (AIST) in Japan. She received her B.S., M.S., and Ph.D. Degrees in Computer Science from Pusan National University in 1998 , 2001, and 2007, respectively. She was a researcher of NICT in Japan from Nov. 2007 to Mar. 2014 and a visiting researcher at the NIST, USA in 2012. Her research interests are in the areas of GIS, Spatiotemporal databases, especially the integration and management

of Sensor and Social data, and Cloud computing. She is the Best Paper Award winner of W2GIS 2009 and the Best Demonstration Runner-up of ACM SIGSPATIAL GIS 2011. Dr. Kim served as PC Co-Chair of W2GIS 2011 and has been serving as a program committee member for IEEE MDM, W2GIS, DBKDA, etc. 